\title{
Estudo do comportamento reológico de polpas de caju (Anacardium occidentale, L.), acerola (Malpighia emarginata, D.C.) e manga (Mangifera indica, L.)
}

\section{Study of rheological behavior of cashew apple (Anacardium occidentale, L.), acerola (Malpighia emarginata, D.C.) and mango (Mangifera indica, L.) pulps}

\author{
Larissa Morais Ribeiro da Silva ${ }^{1 *}$; Geraldo Arraes Maia ${ }^{2}$; \\ Raimundo Wilane de Figueiredo ${ }^{3}$; Paulo Henrique Machado de Sousa ${ }^{4}$; \\ Maria Leônia da Costa Gonzaga ${ }^{5}$; Evânia Altina Teixeira de Figueiredo ${ }^{6}$
}

\section{Resumo}

O conhecimento do comportamento reológico das polpas de frutas tropicais é muito importante para os consumidores e para a indústria de alimentos. Neste trabalho se estudou o comportamento reológico de polpas de caju (Anacardium occidentale, L.), manga (Mangifera indica, L) e acerola (Malpighia emarginata, D.C.) integrais, pasteurizadas e congeladas. As medidas experimentais foram realizadas em reômetro rotacional de cilindros concêntricos e os dados experimentais foram ajustados aos modelos de Ostwald-de-Waelle e Bingham. Também foram avaliados os parâmetros químicos, físico-químicos e microbiológicos. No estudo, para o ajuste dos parâmetros reológicos, o modelo que apresentou maior ajuste foi o de Ostwald-de-Waelle para as polpas de acerola e manga e Bingham para a polpa de caju. As amostras apresentaram comportamento não-newtoniano e caráter pseudoplástico. As caracterizações químicas e físico-químicas demonstraram a riqueza nutricional dos frutos estudados e satisfatórias condições higiênico-sanitárias no processamento realizado nas polpas.

Palavras-chave: Polpa de caju, polpa de acerola, polpa de manga, análise reológica

\begin{abstract}
The knowledge of the fruit tropical pulps rheological behavior have a great importance for the consumers and food process industries. In this work it was studied the rheological behavior of the cashew apple (Anacardium occidentale, L.), mango (Mangifera indica, L) and acerola (Malpighia emarginata, DC) single strength, frozen and pasteurized pulps. The experimental measurements were carried out in concentric cylinder rheometer and the experimental data were fitted to models of Ostwald-de-Waelle and Bingham. There were evaluated chemical, physical-chemical and microbiological parameters. In this work, in order to adjust the rheological parameters, the model that presented the best description was the Ostwald-de-Waelle for the mango and acerola pulps, and Bingham for cashew apple pulp.
\end{abstract}

\footnotetext{
${ }^{1}$ Doutoranda do curso de Ciência e Tecnologia de Alimentos, Universidade Federal do Ceará, UFC, Ceará, CE. E-mail: larissamrs@yahoo.com.br

2 Prof. PhD Emérito, Dept ${ }^{\circ}$ de Tecnologia de Alimentos, UFC, Ceará, CE. E-mail: gmaia@secrel.com

3 Prof. Dr. do Dept ${ }^{\circ}$ de Tecnologia de Alimentos, UFC, Ceará, CE. E-mail: figueira@ufc.br

4 Prof. Dr. do Instituto de Cultura e Arte, UFC, Ceará, CE. E-mail: phenriquemachado@gmail.com

5 Pós-doutoranda em Ciência e Tecnologia de Alimentos, UFC, Ceará, CE. E-mail: leonia_gonzaga@yahoo.com.br

${ }^{6}$ Prof $^{\mathrm{a}}$ Dr $^{\mathrm{a}}$ do Dept ${ }^{\mathrm{o}}$ de Tecnologia de Alimentos, UFC, Ceará, CE. E-mail: evania@ufc.br

* Autor para correspondência
} 
The samples presented non-Newtonian and pseudoplastic character. The chemical and physicochemical analysis showed the nutritional value of the studied fruits. The microbiological results presented satisfactory hygienic and sanitary conditions in pulps processing.

Key words: Cashew-apple pulp, acerola pulp, mango pulp, rheological measurement

\section{Introdução}

Com uma extensão territorial de 8.512.965 $\mathrm{km}^{2}$, o Brasil produz 43 milhões de toneladas de frutas tropicais, subtropicais e de clima temperado, proporcionando ao país uma grande diversidade de frutas o ano inteiro. Dessa forma, o Brasil se destaca na produção de frutas frescas e processadas, sendo considerado o terceiro maior produtor mundial de frutas, perdendo apenas para China e Índia (IBRAF, 2010).

O consumo de sucos de frutas no país encontrase em plena expansão em várias regiões. Várias frutas dessas regiões apresentam composição em aroma e compostos funcionais de grande valor (OLIVEIRA et al., 2009; SOUSA et al., 2007), como é o caso do caju (Anacardium occidentale, L.), manga (Mangifera indica, L.) e acerola (Malphigia emarginata, D.C.).

$\mathrm{O}$ caju, dentre as frutas tradicionais, destaca-se como boa fonte de pró-vitamina A, além de possuir excelentes qualidades de sabor e aroma (MAIA; SOUSA; LIMA, 2007). O caju é de interesse nutricional por apresentar, principalmente, um elevado teor de vitamina $\mathrm{C}$, apresentando para o pedúnculo maduro teores que variam de 180 a 250 $\mathrm{mg} / 100 \mathrm{~mL}$ de suco, compostos fenólicos, vitamina do complexo B, minerais e pigmentos carotenóides (FIGUEIREDO et al., 2007).

A acerola apresenta grande potencial econômico e nutricional, considerada uma fruta bastante utilizada em diversos produtos visando o incremento de vitamina C (PEREIRA et al., 2009; ARAÚJO et al., 2009), associado também à presença de carotenóides e antocianinas, destacando-se no campo dos alimentos funcionais (FREITAS et al., 2006).
A manga é originária do sudeste da Ásia e da Índia, possui sabor acentuado e característico (DAK; VERMA; SHARMA, 2006), sendo consumida principalmente na forma in natura e tem na industrialização uma excelente alternativa para manutenção de seus atributos de qualidade (OLIVEIRA, 2006), apresentando-se como boa fonte de antioxidantes para a dieta humana, com boas quantidades de carotenóides e compostos fenólicos (RIBEIRO et al., 2007).

Consumidores mais exigentes e com maior poder de compra têm estimulado estudos com vistas ao aperfeiçoamento dos processos e melhorias da qualidade dos produtos agrícolas, resultando na adaptação de sistemas e equipamentos industriais. No processamento industrial de polpas, doces e demais derivados de frutas em estado fluido ou pastoso, os estudos das propriedades reológicas têm aplicação fundamental (GRANJEIRO et al., 2007).

No Brasil, são poucos os trabalhos realizados a fim de determinar as propriedades físico-químicas e reológicas de polpas das frutas nacionais. Essas propriedades são úteis não só como medida de qualidade, mas também no projeto dos processos de industrialização, tais como bombeamento, agitação, transporte em tubulações e evaporação (BRANCO, 2001).

Este trabalho teve como objetivo avaliar o comportamento reológico, além dos parâmetros microbiológicos, químicos e físico-químicos $(\mathrm{pH}$, sólidos solúveis totais, acidez titulável, açucares redutores e torais, antocianinas torais, vitamina $\mathrm{C}$, polifenóis extraíveis totais e cor) de polpas de caju, manga e acerola. 


\section{Materiais e Métodos}

\section{Matéria-prima}

Neste trabalho foram utilizadas polpas de caju, acerola, e manga, pasteurizadas e congeladas, fornecidas por uma indústria de polpas de frutas localizada em Pacajus/CE, estando embaladas em sacos plásticos de polietileno com capacidade de $2000 \mathrm{~mL}$. As amostras encontravam-se dentro do prazo de validade e foram descongeladas sob refrigeração, homogeneizadas e submetidas imediatamente às determinações analíticas.

\section{Análise reológica}

Tendo em vista o alto teor de fibras encontrado na polpa de caju, para a realização das análises reológicas, inicialmente, as amostras congeladas dessa fruta foram submetidas a um homogeneizador OMNI, a $10000 \mathrm{rpm}$ por um minuto, a fim de diminuir o tamanho das partículas. O mesmo tratamento foi dado às polpas de acerola e manga.

O comportamento reológico das polpas de caju, acerola e manga foi determinado através de um reômetro rotacional de cilindros concêntricos tipo Searle da Brookfield, modelo R/S plus SST 2000. As medidas foram feitas na temperatura de $25^{\circ} \mathrm{C}$, a qual foi ajustada através de um banho termostático acoplado ao equipamento. $\mathrm{O}$ equipamento forneceu os dados de tensão de cisalhamento e taxa de deformação através do software RHEO V2. 8. As análises reológicas foram obtidas com variação da taxa de deformação de 0 a $500 \mathrm{~s}^{-1}$ (curva ascendente)

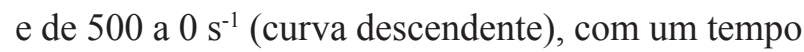
de 1 minuto e leitura de 25 pontos para cada curva. As leituras foram feitas em triplicata e em cada medida foi utilizada uma nova amostra.

\section{Caracterização química e físico-química}

$\mathrm{O} \mathrm{pH}$ foi determinado através de leitura direta, em potenciômetro de marca WTW, modelo 330i/ SET (Quimis - São Paulo), calibrado a cada utilização, com soluções tampão de $\mathrm{pH}$ 4,0 e 7,0 conforme AOAC (1995). A determinação dos sólidos solúveis totais foi realizada por refratometria através da medida dos Brix, em refratômetro marca ATAGO (EUA), com escala variando de 0 a 90 Brix, conforme Brasil (2005). Os açúcares redutores e totais foram feitos conforme Miller (1959). Para a determinação dos açúcares não redutores foi realizada uma inversão ácida prévia nos extratos das amostras (BRASIL, 2005). A determinação da acidez titulável foi realizada por titulometria, utilizando-se $1 \mathrm{~mL}$ de amostra. A titulação foi feita com uma solução de $\mathrm{NaOH} 0,1 \mathrm{M} / \mathrm{L}$, segundo a técnica descrita pela AOAC (1995), e fenolftaleína $1 \%$ como indicador, sendo o resultado expresso em $\mathrm{g}$ de ácido cítrico/100mL de amostra. Os teores de antocianinas totais foram determinados segundo a metodologia descrita por Francis (1982), onde foi homogeneizado $1 \mathrm{~mL}$ da amostra com solução de $\mathrm{HCl} 1,5 \mathrm{M}$ e etanol $85 \%$, v/v para sua extração. Esse homogeneizado foi submetido a uma noite de repouso sob refrigeração e na ausência de luz. A seguir o extrato foi filtrado e feita a leitura do mesmo a $535 \mathrm{~nm}$, sendo os resultados expressos em $\mathrm{mg} / 100$ $\mathrm{mL}$. O conteúdo de vitamina $\mathrm{C}$ foi determinado por meio do método titulométrico baseado na redução do indicador 2,6-diclorofenolindofenol pelo ácido ascórbico (PEARSON, 1976), sendo os resultados expressos em mg de ácido ascórbico/100 $\mathrm{mL}$ de amostra. Os polifenóis extraíveis totais foram determinados de acordo com a metodologia descrita por Reicher, Sierakowski e Corrêa (1981), usando o reagente de Folin-Ciocalteu, tendo o ácido tânico como padrão, sendo a leitura feita em espectrofotômetro UV-1800 (Japão) a $760 \mathrm{~nm}$. A determinação da cor instrumental foi feita pela média de leituras efetuadas em uma placa de petri, sendo usado quantidade suficiente de amostra para cobrir a base da placa. As medidas foram realizadas em colorímetro Konica Minolta spectrophotometer CM - 3500d (Japão). Os resultados foram expressos de acordo com as coordenadas CIE lab, que inclui as variáveis $L^{*}, a^{*}$ e b. As análises foram realizadas em triplicata. 
Análises microbiológicas

Foram realizadas análises de Salmonella/25 g, contagem de coliformes a $45{ }^{\circ} \mathrm{C}$ e $35{ }^{\circ} \mathrm{C}(\mathrm{NMP} / \mathrm{g})$, contagem de bactérias aeróbias mesófilas (UFC/g) e contagem de bolores e leveduras (UFC/g), seguindo metodologia descrita por Silva, Junqueira e Silveira (2001).

\section{Análise estatística}

Para as análises reológicas, os dados obtidos foram ajustados aos modelos de Ostwald-de-Waelle e Bingham, por análise de regressão não linear, através do software Statistica 7.0.

\section{Resultados}

Os parâmetros reológicos do modelo de Ostwaldde-Waelle, K (índice de consistência) e $n$ (índice de comportamento do fluido), são apresentados na Tabela 1. A polpa de caju apresentou maior valor de K $(2,2963)$ e poucas variações para os valores de $n$ $(0,3391$ a 0,3760$)$.

Para os parâmetros decorrentes do modelo de Bingham (Tabela 2), maior viscosidade aparente $\left(\eta_{\mathrm{pl}}\right)$ e tensão inicial $\left(\tau_{0}\right)$ foram obtidos para a polpa de manga.

$\mathrm{O}$ intervalo de valores de $\mathrm{R}^{2}$ (Coeficiente de determinação), de 0,8437 a 0,9871 , para os dois modelos trabalhados, indicaram bons ajustes a esses modelos.

Tabela 1. Parâmetros reológicos do modelo de Ostwald-de-Waelle K (Índice de consistência), n (Índice de comportamento do fluido) e $\mathrm{R}^{2}$ (Coeficiente de determinação) para polpas de caju (Anacardium occidentale, L.), acerola (Malpighia emarginata, D.C.) e manga (Mangifera indica, L.), integrais pasteurizadas e congeladas.

\begin{tabular}{cccc}
\hline Polpas integrais, & \multicolumn{3}{c}{ Parâmetros reológicos } \\
\cline { 2 - 4 } pasteurizadas e congeladas & $\mathrm{K}$ & $\mathrm{n}$ & $\mathrm{R}^{2}$ \\
\hline Caju & 2,2963 & 0,3391 & 0,8437 \\
Acerola & 1,3779 & 0,3514 & 0,9871 \\
Manga & 1,3878 & 0,3760 & 0,9716 \\
\hline
\end{tabular}

Tabela 2. Parâmetros reológicos do modelo de Bingham $\eta_{\mathrm{pl}}$ (viscosidade aparente), $\tau_{0}$ (tensão inicial) e $\mathrm{R}^{2}$ (Coeficiente de determinação) para as polpas de caju (Anacardium occidentale, L.), acerola (Malpighia emarginata, D.C.) e manga (Mangifera indica, L.), integrais pasteurizadas e congeladas.

\begin{tabular}{cccc}
\hline \multirow{2}{*}{$\begin{array}{c}\text { Polpas integrais pasteurizadas e } \\
\text { congeladas }\end{array}$} & \multicolumn{3}{c}{ Parâmetros reológicos } \\
\cline { 2 - 4 } & $\eta_{\mathrm{pl}}$ & $\tau_{0}$ & $\mathrm{R}^{2}$ \\
\hline Caju & 0,0175 & 4,5525 & 0,9337 \\
Acerola & 0,0174 & 4,2555 & 0,9337 \\
Manga & 0,0209 & 5,0922 & 0,9363 \\
\hline
\end{tabular}

O comportamento gráfico pode ser observado na Figura 1, onde é verificado aumento da tensão de cisalhamento com o aumento da taxa de deformação, comportamento característico de fluido nãonewtoniano do tipo pseudoplástico, cujos valores de (n) (Tabela 1), menores que um, reforçam tal classificação. 


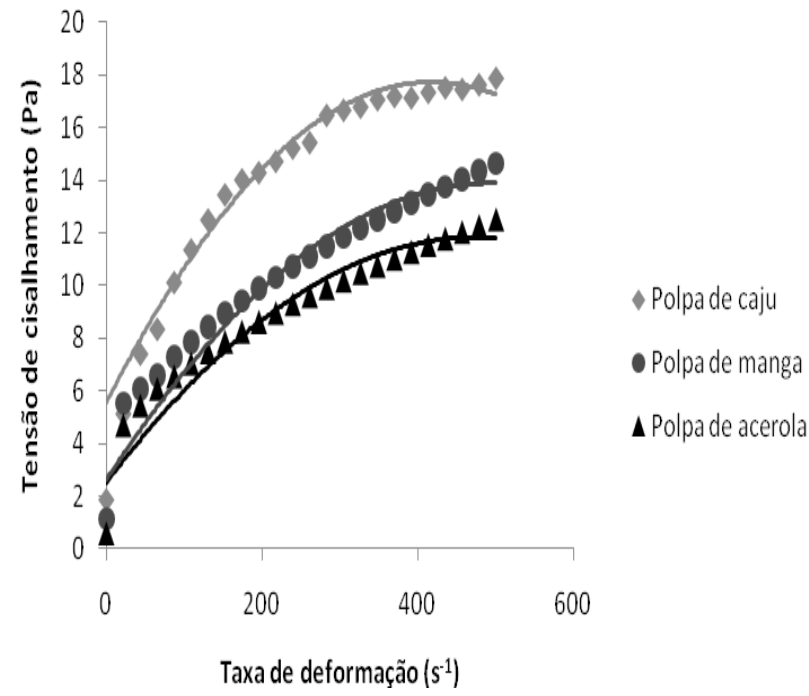

Figura 1. Relação entre tensão de cisalhamento e taxa de deformação para polpas de caju (Anacardium occidentale, L.), acerola (Malpighia emarginata, D.C.) e manga (Mangifera indica, L.), integrais pasteurizadas e congeladas.

Os resultados das análises químicas e físicoquímicas das polpas de caju, acerola e manga são apresentados na Tabela 3.

Pouca variação foi observada para os valores de $\mathrm{pH}(3,73$ a 3,92). Os sólidos solúveis totais apresentaram valores de 5,80 e 14,9 ${ }^{\circ}$ Brix, para as polpas de acerola e manga, respectivamente. A acidez titulável se destacou para a polpa de acerola com $0,81 \mathrm{~g}$ de ácido cítrico/100g. O teor de açúcar redutor na polpa de caju $(7,43 \%)$ se mostrou bem maior que a média de $2,85 \%$ determinada para as demais polpas. Na polpa de manga foi determinado maior teor de açúcar total, 31,29\%. Grandes variações foram encontradas nos teores de antocianinas totais, com valores de 47,74 a 2051,62 $\mathrm{mg} / 100 \mathrm{~mL}$. Como esperado a polpa de acerola apresentou maior teor de vitamina C (770,73 mg de ácido ascórbico/100g). Rico conteúdo de polifenóis totais foi determinado para a polpa de acerola (1956,53mg/100g). Para os parâmetros de cor, em análise geral, não foi observado grandes variações nos valores determinados, apresentando-se a luminosidade ( $\mathrm{L}^{*}$ ) com valores de 37,63 a 43,51, os valores de $a^{*}$ de $-1,40$ a 2,93 e b* com valores de 10,44 a 14,46 .

As análises microbiológicas (Tabela 4) mostraram ausência de Salmonella/25g e valores $<3 \mathrm{NMP} / \mathrm{g}$ para Coliformes a $45^{\circ} \mathrm{C}$ e $35^{\circ} \mathrm{C}$, para todas as polpas. Bactérias aeróbias mesófilas foram identificadas em valores de UFC/g de 1,9x10² a $2,5 \times 10^{3}$. Para bolores e leveduras foram observados valores de $10 \mathrm{UFC/g}$ para as polpas de caju e acerola $\mathrm{e}<10 \mathrm{UFC} / \mathrm{g}$ para a polpa de manga.

Tabela 3. Resultados das análises químicas e físico-químicas das polpas de caju (Anacardium occidentale, L.), acerola (Malpighia emarginata, D.C.) e manga (Mangifera indica, L.), integrais pasteurizadas e congeladas.

\begin{tabular}{|c|c|c|c|}
\hline Análise & Polpa de Caju & Polpa de Acerola & Polpa de Manga \\
\hline $\mathrm{pH}$ & $3,92 \pm 0,02$ & $3,73 \pm 0,06$ & $3,85 \pm 0,06$ \\
\hline Sólidos Solúveis totais ( $\left.{ }^{\circ} \mathrm{Brix}\right)$ & $10,47 \pm 0,06$ & $5,80 \pm 0,00$ & $14,90 \pm 0,00$ \\
\hline Acidez titulável (g de ácido cítrico/100g) & $0,57 \pm 0,14$ & $0,81 \pm 0,53$ & $0,57 \pm 0,14$ \\
\hline Açúcar redutor $(\%)$ & $7,43 \pm 1,61$ & $2,86 \pm 0,09$ & $2,87 \pm 0,09$ \\
\hline Açúcar total $(\%)$ & $7,82 \pm 0,73$ & $12,13 \pm 3,77$ & $31,29 \pm 2,90$ \\
\hline Antocianinas totais $(\mathrm{mg} / 100 \mathrm{~mL})$ & $47,74 \pm 47,55$ & $2051,62 \pm 34,97$ & $126,13 \pm 1,68$ \\
\hline Vitamina C (mg de ácido ascórbico/100g) & $96,55 \pm 2,69$ & $770,73 \pm 5,11$ & $20,88 \pm 0,89$ \\
\hline Polifenóis extraíveis totais & $844,36 \pm 60,54$ & $1956,53 \pm 29,38$ & $193,12 \pm 9,29$ \\
\hline \multirow[t]{3}{*}{$\mathrm{L}^{*}$} & $43,51 \pm 0,14$ & $37,63 \pm 0,15$ & $39,23 \pm 0,26$ \\
\hline & $-1,40 \pm 0,02$ & $2,93 \pm 0,08$ & $1,08 \pm 0,22$ \\
\hline & $12,98 \pm 0,38$ & $10,44 \pm 0,11$ & $14,46 \pm 0,79$ \\
\hline
\end{tabular}

Médias e Desvio padrão. 
Tabela 4. Resultados das análises microbiológicas das polpas de caju (Anacardium occidentale, L.), acerola (Malpighia emarginata, D.C.) e manga (Mangifera indica, L.), integrais pasteurizadas e congeladas.

\begin{tabular}{lccc}
\hline \multicolumn{1}{c}{ Análise } & Polpa de Caju & Polpa de Acerola & Polpa de Manga \\
\hline Salmonella sp. & Ausência/25g & Ausência/25g & Ausência/25g \\
Coliformes a $45^{\circ} \mathrm{C}(\mathrm{NMP} / \mathrm{g})$ & $<3 \mathrm{NMP} / \mathrm{g}$ & $<3 \mathrm{NMP} / \mathrm{g}$ & $<3 \mathrm{NMP} / \mathrm{g}$ \\
Coliformes a $35^{\circ} \mathrm{C}(\mathrm{NMP} / \mathrm{g})$ & $<3 \mathrm{NMP} / \mathrm{g}$ & $<3 \mathrm{NMP} / \mathrm{g}$ & $<3 \mathrm{NMP} / \mathrm{g}$ \\
Bactérias aeróbias mesófilas (UFC/g) & $1,9 \times 10^{3}$ & $2,5 \times 10^{3}$ & $1,9 \times 10^{2}$ \\
Bolores e leveduras (UFC/g) & 10 & 10 & $<10$ \\
\hline
\end{tabular}

NMP- Número mais provável

UFC- unidade formadora de colônias.

\section{Discussão}

Os parâmetros reológicos obtidos através do ajuste dos dados de tensão de cisalhamento e taxa de deformação aos modelos de Ostwald-de-Waelle e Bingham podem ser observados nas Tabelas 1 e 2, respectivamente.

Os dados obtidos na análise reológica para as polpas de acerola e manga foram melhores ajustados ao modelo de Ostwald-de-Waelle, onde foi observado maior valor de coeficiente de determinação $(R 2>0,9716)$, enquanto para a polpa de caju os dados obtiveram melhor ajuste ao modelo de Bingham $(\mathrm{R} 2=0,9337)$. A polpa de caju necessita de uma tensão de cisalhamento inicial para escoar, o que reflete no melhor ajuste ao modelo de Bingham (Figura 1).

Analisando o comportamento reológico das polpas de caju, manga e acerola (Figura 1), a polpa de caju apresentou, com o aumento da taxa de deformação, maior tensão de cisalhamento quando comparada com as demais polpas estudadas, seguida das polpas de manga e acerola. Esse fato pode ser justificado pela grande presença de fibras na polpa de caju, que pode fornecer a mesma característica bastante viscosa.

Nos ensaios reológicos realizados, em sentido ascendente e descendente com a taxa de deformação, não foram observados efeitos de histerese, o que significa que as polpas analisadas não apresentaram comportamento dependente do tempo (tixotropia).

As polpas de caju, acerola e manga apresentaram comportamento não-newtoniano, com índices de comportamento (n) menores que a unidade, representando comportamento pseudoplástico.

De acordo com Sugai (2002), as polpas de frutas, dispersões de moléculas ou partículas assimétricas, apresentam no repouso um estado desordenado e quando submetidas a uma tensão de cisalhamento, suas moléculas ou partículas tendem a orientar-se na direção da força aplicada. Quanto maior a força aplicada, maior será a ordenação, e conseqüentemente, menor a viscosidade aparente. Como a viscosidade aparente das polpas de frutas decresce com o aumento da tensão de cisalhamento, estas são classificadas como fluidos pseudoplásticos.

Em trabalho realizado por Vidal (1997) sobre o comportamento reológico do suco de manga, encontrou-se comportamento pseudoplástico tanto para o suco integral, como centrifugado e despectinizado, assim como em estudo realizado com polpa de manga centrifugada (VIDAL et al., 2006).

Dak, Verma e Jaaffrey (2007) estudaram o comportamento de suco de manga da variedade "Totapuri", e observaram comportamento pseudoplástico, semelhante ao observado para o suco de manga da variedade Kesar, analisado por Dak, Verma e Sharma (2006).

Silva, Guimarães e Gasparetto (2005) analisaram o comportamento reológico do suco de acerola em concentrações de sólidos solúveis de $4-16$ ${ }^{\circ}$ Brix e no intervalo de temperatura de $5-85,0$ ${ }^{\circ} \mathrm{C}$ e constataram um forte comportamento não- 
newtoniano com características pseudoplásticas. O comportamento da amostra aqui estudada se assemelha as divulgadas.

Para a polpa de caju, em relação à análise de $\mathrm{pH}$ o resultado obtido foi de 3,92, o qual é próximo ao valor encontrado por Gadelha et al. (2009), ao avaliarem polpa congelada de caju, obtendo valor de $\mathrm{pH}$ de 3,85 .

Para a polpa de manga o $\mathrm{pH}$ obtido foi de 3,85 . Benevides et al. (2008), analisando polpa de manga Ubá, obtiveram valores de $\mathrm{pH}$ variando entre 4,12 a 4,29 , resultados próximos aos obtidos neste trabalho. Valores de $\mathrm{pH}$ próximos, 4,3 e 3,85, também foram encontrados por Faraoni, Ramos e Stringheta (2009) e Akhtar et al. (2010), respectivamente.

Obteve-se $\mathrm{pH}$ de 3,73 para polpa de acerola. Matssura et al. (2001) analisando acerolas, obtiveram $\mathrm{pH}$ variando na faixa entre 3,18 a 3,44, valores inferiores aos obtidos neste trabalho, assim como Gadelha et al. (2009), que obteve pH de 2,85 para polpa congelada de acerola.

Em relação aos sólidos solúveis totais, a polpa de acerola foi a que apresentou menor teor quando comparada às demais polpas estudadas, apresentando 5,80 Brix. Gadelha et al. (2009) obtiveram teor de sólidos solúveis totais de 8,77 para polpa de acerola congelada. Matssura et al. (2001) analisaram frutos de diferentes genótipos de acerola e obtiveram teores de sólidos solúveis totais variando de 6,0 a 11,4 Brix. Araújo et al. (2009), avaliaram diferentes clones de acerola, obtendo teores de sólidos solúveis variando de 6,8 a 7,1.

Scherer, Rybka e Godoy (2008) analisaram o teor de ácido orgânico em polpa de caju, obtendo uma média de 0,61 g de ácido cítrico/100 g de polpa, resultado próximo ao observado neste estudo (Tabela 3).

Para a polpa de manga, em relação à análise de acidez, obteve-se teor de $0,57 \mathrm{~g}$ de ácido cítrico/100 $\mathrm{g}$ de polpa. Benevides et al. (2008) analisando polpa de manga Ubá obteve valores de acidez variando entre 0,44 e 0,63 mg de ácido cítrico/100 g de polpa. Jeronimo et al. (2007) constataram teor de acidez total tituláve1 de 1,09 g/100 g de polpa de manga. Os valores obtidos neste trabalho encontraram-se dentro da faixa especificada por estes autores. Dentre os frutos estudados, a acerola apresentou maior acidez, apresentando $0,80 \mathrm{~g}$ de ácido cítrico/100 g de polpa.

Scherer, Rybka e Godoy (2008) analisaram o teor de ácido ascórbico em polpa de caju, obtendo valor de 113,37 mg de ácido ascórbico/100 g de polpa, resultado semelhantes ao obtido nesse trabalho (Tabela 1). Assunção e Mercadante (2003) e Pereira et al (2008), analisando polpas de caju, obtiveram teores de 56,6 a 117,6 e 43,1 a 152 mg de ácido ascórbico/100 g de polpa, respectivamente, sendo valores semelhantes aos encontrados na polpa em estudo. As polpas de acerola e manga apresentaram teores de vitamina $\mathrm{C}$ de 770,70 e $20,88 \mathrm{mg}$ de ácido ascórbico/100 g, respectivamente. Yamashita, Benassi e Tonzar (2003), analisando polpa de acerola congelada, obtiveram teor de vitamina $\mathrm{C}$ de $1511 \mathrm{mg} / 100 \mathrm{~g}$, valor cerca $50 \%$ mais elevado que o obtido nesse trabalho.

Para a análise de açúcares, as polpas de caju, acerola e manga apresentaram respectivamente, $7,43 \%, 2,86 \%$ e 2,87\% para açúcares redutores e $7,82 \%, 12,13 \%$ e $31,29 \%$ para açúcares totais. Faraoni, Ramos e Stringheta (2009) avaliaram as características físico-químicas de polpa de manga orgânica, obtendo para a análise de açúcares totais teor de $10,5 \%$, valor inferior ao obtido nesse trabalho, e para açúcares redutores teor de 5\%, valor superior ao obtido nesse estudo. Maia et al. (2004) analisando polpa de caju, obtiveram teores de açúcares redutores e totais de 8,30 e 8,74, respectivamente, resultados próximos aos obtidos neste estudo.

Para a análise de antocianinas, a polpa de acerola apresentou maior teor $(2051,62 \mathrm{mg} / 100 \mathrm{~g})$, seguida das polpas de manga $(126,13 \mathrm{mg} / 100 \mathrm{~g})$ e caju $(47,74 \mathrm{mg} / 100 \mathrm{~g})$. 
Lima et al. (2000) pesquisaram os teores de antocianinas em acerola, obtendo uma variação de 14,06 a $50,98 \mathrm{mg}$ de antocianinas $/ 100 \mathrm{~g}$, resultados consideravelmente inferiores aos obtidos nesse estudo. Essa diferença dos resultados obtidos para esta análise quando comparada aos resultados obtidos por outros autores pode ser devido às antocianinas serem pigmentos muito instáveis, podendo facilmente ocorrer a degradação (LIMA et al., 2003).

Silva (2008), estudando frutos maduros de acerola, encontrou resultados que variaram de $3,87 \mathrm{mg} / 100 \mathrm{~g}$ a $21,55 \mathrm{mg} / 100 \mathrm{~g}$. Aguiar (2001) encontrou conteúdo de antocianinas totais variando de 0,37 a $38,38 \mathrm{mg} / 100 \mathrm{~g}$ de polpa.

Melo et al. (2008) obtiveram teores de fenólicos totais de 409,25; 90,44 e 20951,79 mg em equivalente de catequina, em $100 \mathrm{~g}$ de polpas de caju, manga e acerola, respectivamente.

As análises revelaram teores de polifenóis extraíveis totais de $1956,53 \mathrm{mg} / 100 \mathrm{~g}$ de acerola e $193,12 \mathrm{mg} / 100 \mathrm{~g}$ de manga. As medidas demonstraram, para todas as polpas de fruta estudadas, valores de b acima de 10. Dessa forma, como os valores obtidos foram positivos, as polpas de acerola, caju e manga apresentaram maior tendência para uma coloração amarelada. Para o parâmetro de cor "a", apenas a polpa de caju apresentou valores de a negativos, apresentando maior tendência para uma coloração esverdeada. Todas as amostras apresentaram valores de luminosidade acima de 30 .

Benevides et al. (2008) obtiveram para os parâmetros de cor L*, a* e b*, respectivamente 57,99, 15,91 e 49,11, para polpa de manga Ubá. Resultados superiores aos obtidos nesse trabalho, representando maior luminosidade e coloração mais próxima das cores vermelham $\left(a^{*}\right)$ e amarela $\left(b^{*}\right)$.

Em relação aos padrões microbiológicos sanitários, a legislação brasileira estabelece para polpas de frutas ausência de Salmonella sp. em 25 g e limite máximo de contagem de coliformes fecais de $10^{2}$ UFC ou NMP/g (BRASIL, 2001). Os resultados das análises microbiológicas para os frutos estudados são apresentados na Tabela 4.

Os resultados de Salmonella sp. e coliformes a 45 ${ }^{\circ} \mathrm{C}$ demonstram a inocuidade das polpas analisadas, estando de acordo com os padrões exigidos pela legislação vigente.

As baixas contagens de bactérias aeróbias mesófilas (UFC/g) e bolores e leveduras (UFC/g) (Tabela 4) indicam a eficiência do processo de pasteurização bem como uma boa condição higiênica da indústria processadora.

Feitosa et al. (1997) estudaram o perfil microbiológico de polpas de acerola e caju congeladas, obtendo contagens de coliformes a 45 ${ }^{\circ} \mathrm{C}$ e $35{ }^{\circ} \mathrm{C}<3 \mathrm{NMP} / \mathrm{g}$ e ausência de Salmonella $s p / 25 \mathrm{~g}$ em todas as amostras analisadas. Para polpas de acerola obtiveram contagens de bactérias aeróbias mesófilas variando entre $1,0 \times 10^{3}$ a $7,9 \times 10^{3} \mathrm{UFC} / \mathrm{g}$ de polpa e para bolores e leveduras, contagens variando entre $<10$ a $8,9 \times 10^{3} \mathrm{UFC} / \mathrm{g}$ de polpa. Em polpas de caju foram obtidas contagens de bactérias aeróbias mesófilas variando entre $<10$ UFC/g a 5,0x $10^{1} \mathrm{UFC} / \mathrm{g}$ de polpa e para a análise de bolores e leveduras, os autores obtiveram contagens variando entre $<10$ a $8,1 \times 10^{3} \mathrm{UFC} / \mathrm{g}$ de polpa.

Santos, Coelho e Carreiro (2008) realizaram análises microbiológicas de polpas de acerola e caju, onde obtiveram para a polpa de acerola contagens de bolores e leveduras com valor mínimo $<10$ $\mathrm{UFC} / \mathrm{g}$ e máximo de $2,3 \times 10^{4} \mathrm{UFC} / \mathrm{g}$ de polpa. Para a polpa de caju, os autores obtiveram contagens de bolores e leveduras variando entre um mínimo correspondente a $5,0 \times 10^{2} \mathrm{UFC} / \mathrm{g}$ e um máximo de $6,2 \times 10^{4} \mathrm{UFC} / \mathrm{g}$.

\section{Conclusões}

As polpas de caju, manga e acerola apresentaram comportamento não-newtoniano e caráter pseudoplástico, obtendo-se melhor ajuste para os dados referentes às polpas de acerola e manga ao modelo de Ostwald-de-Waelle e para polpa de caju ao modelo de Binhgam. 
As análises químicas e físico-químicas demonstraram a riqueza nutricional em componentes funcionais das polpas integrais, pasteurizadas e congeladas estudadas. As polpas de acerola e caju apresentaram elevados teores de vitamina $\mathrm{C}$ e polifenóis extraíveis totais, seguida da polpa de manga, que apresentou elevado teor de antocianinas totais, juntamente com a polpa de acerola. As polpas estudadas apresentaram condições higiênicosanitárias satisfatórias.

\section{Referências}

AGUIAR, L. P. $\beta$-Caroteno, vitamina $C$ e outras características de qualidade de acerola, caju e melão em utilização no melhoramento genético. 2001. Dissertação (Mestrado em Tecnologia de Alimentos) - Universidade Federal do Ceará, Fortaleza.

AKHTAR, S.; RIAZ, M.; AHMAD, A.; NISAR, A. Physico-chemical, microbiological and sensory stability of chemically preserved mango pulp. Pak. J. Bot., Paquistão, v. 42, n. 2, p. 853-862, 2010.

ASSOCIATION OF OFFICIAL ANALITICAL CHEMISTRY - AOAC. Official methods of analysis of the association of official analytical chemistry. 16. ed. Washington, D. C. 1995.1141 p.

ARAÚJO, P. G. L.; FIGUEIREDO, R. W.; ALVES, R. E.; MAIA, G. A.; MOURA, C. F. H.; SOUSA, P. H. M. Qualidade físico-química de frutos de clone de aceroleira recobertos com filme de PVC e conservados por refrigeração. Semina: Ciências Agrárias, Londrina, v. 30, n. 4, p. 867-880, 2009.

ASSUNÇÃO, R. B.; MERCADANTE, A. Z. Carotenoids and ascorbic acid composition from commercial products of cashew apple (Anacardium occidentale, L.). J Food Compos Anal, Estados Unidos, v. 16, n. 6, p. 647-657, 2003.

BENEVIDES, S. D.; RAMOS, A. M.; STRINGUETA, P. C.; CASTRO, V. C. Qualidade da manga e polpa da manga ubá. Ciência e Tecnologia de Alimentos, Campinas, v. 28, n. 3, p. 571-578, 2008.

BRANCO, I. G. Estudo do comportamento reológico de misturas ternárias com sucos de manga, laranja e cenoura. 2001. Tese (Doutorado em Engenharia de Alimentos) - Universidade Estadual de Campinas, Campinas.

BRASIL. Ministério da Saúde. Agência Nacional de Vigilância Sanitária. Métodos físico-químicos para análise de alimentos. Ministério da Saúde, Agência Nacional de Vigilância Sanitária. Brasília: Ministério da Saúde, 2005. 1018 p.

$\mathrm{RDC} \mathrm{n}^{\circ} 12$, de $02 / 01 / 2001$. Regulamento técnico sobre os padrões microbiológicos para alimentos. Diário Oficial da República Federativa do Brasil, Brasília. 2001.

DAK, M.; VERMA, R. C.; JAAFFREY, S. N. A. Effect of temperature and concentration on rheological properties of "Kesar" mango juice. Journal of Food Engineering, Essex, v. 80, n. 4, p. 1011-1015, 2007.

DAK, M.; VERMA, R. C.; SHARMA, G. P. Flow characteristics of juice of "Totapuri" mangoes. Journal of Food Engineering, Essex, v. 76, n. 4, p. 557-561, 2006.

FARAONI, A. S.; RAMOS, A. M.; STRINGHETA, P. C. Caracterização da manga orgânica cultivar Ubá. Revista Brasileira de Produtos Agroindustriais, Campina Grande, v. 11, n. 1, p. 9-14, 2009.

FEITOSA, T.; OLIVEIRA, M. E. B.; BASTOS, M. S. R.; MUNIZ, C. R.; OLIVEIRA, S. C.A. Perfil microbiológico de polpa de frutas produzidas e comercializadas no estado do Ceará e Rio Grande do Norte. Bol.CEPPA, Curitiba, v. 15, n. 1, p. 65-74, 1997.

FIGUEIREDO, R. W.; LAJOLO, F. M.; ALVES, R. E.; FILGUEIRAS, H. A. C.; MAIA, G. A.; SOUSA, P. H. M. Qualidade de pedúnculos de caju submetidos a aplicação pós-colheita de cálcio e armazenados sob refrigeração. Pesquisa Agropecuária Brasileira, Brasília, v. 42, n. 4, p. 475-482, 2007.

FRANCIS, F. J. Analysis of anthocyanins. In: MARKAKIS, P. (Ed.). Anthocyanins as food colors. New York: Academic Press, 1982. p. 181-207.

FREITAS, C. A. S.; MAIA, G. A.; SOUSA, P. H. M.; BRASIL, I. M.; PINHEIRO, A. M. Storage stability of acerola tropical fruit juice obtained by hot fill method. International Journal of Food Science \& Technology, v. 4, n. 10, p. 1216-1221, 2006.

GADELHA, A. J. F.; ROCHA, C. O.; VIEIRA, F. F.; RIBEIRO, G. N. avaliação de parâmetros de qualidade físico-químicos de Polpas congeladas de abacaxi, acerola, cajá e caju. Caatinga, Mossoró, v. 22, n. 1, p. 115-118, 2009.

GRANJEIRO,A.A.; QUEIROZ,A. J. M.; FIGUEIRÊDO, F. M. F.; MATA, M. E. R. M. C. Viscosidades de polpas concentradas de figo da índia. Revista Brasileira de Agrociência, Pelotas, v. 13, n. 2, p. 219-224, 2007.

INSTITUTO BRASILEIRO DE FRUTAS - IBRAF. Fruticultura. 2009. Disponível em: <http://www.ibraf. org.br/imprensa/0901_FrutasBrasileirasAscensao.asp>. Acesso em: 15 jan 2010. 
JERONIMO, E. M.; BRUNINI, M. A.; ARRUDA, M. C.; CRUZ, J. C. S.; FISCHER, I. H.; GAVA, G. J. C. Conservação pos-colheita de mangas Tommy Atkins armazenadas sob atmosfera modificada. Semina: Ciências Agrárias, Londrina, v. 28, n. 3, p. 417-423, 2007.

LIMA, V. L. A.; MELO, E. A.; MACIEL, M. I. S.; LIMA, D. E. S. Avaliação do teor de antocianinas em polpa de acerola congelada proveniente de frutos de 12 diferentes aceroleiras (Malpighia emarginata D.C.), Ciência $e$ Tecnologia de Alimentos, Campinas, v. 23, n. 1, p. 101103, 2003.

LIMA, V. L. A. G.; MÉLO, E. A.; LIMA, L. S.; NASCIMENTO, P. P. Flavonóides em seleções de acerola (Malpighia sp. L). 1- Teor de antocianinas e flavonóis totais. Ciência Rural, Santa Maria, v. 30, n. 6, p. 1063-1064, 2000.

MAIA, G. A; SOUZA FILHO, M. S. M.; FIGUEIREDO, R. W.; BRASIL, I. M. Caracterização química de pedúnculos de diferentes clones de cajueiro anão precoce (Anacardium occidentale, L). Revista Ciência Agronômica, Fortaleza, v. 35, p. 272-278, 2004. Especial.

MAIA, G. A.; SOUSA, P. H. M.; LIMA, A. S. L. Processamento de frutas tropicais. Fortaleza: Edições UFC, 2007.

MATSSURA, F. C. A. U.; CARDOSO, R. L.; FOLEGATTI, M. I. S.; OLIVEIRA, J. R. P.; OLIVEIRA, J. A. B.; SANTOS, D. B. Avaliações físico-químicas em frutos de diferentes genótipos de acerola (Malpighia punicifolia, L.). Revista Brasileira de Fruticultura, Jaboticabal, v. 23, n. 3, p. 602-606, 2001.

MELO, E. A.; MACIEL, M. I. S.; LIMA, V. L. A. G.; ARAÚJO, C. R. Teor de fenólicos totais e capacidade antioxidante de polpas congeladas de frutas. Alimentos $e$ Nutricao, Araraquara, v. 19, n. 1, p. 67-72, 2008.

MILLER, G. L. Use of dinitrosalicilic acid reagent for determination of reducing sugar. Analytical Biochemistry, New York, v. 31, n. 3, p. 426-428, 1959.

OLIVEIRA, A. B. Caracterização físico-química, química e bioquímica do suco tropical de manga (Mangifera indica. L) não adoçado obtido pelo processo hot fill. 2006. Dissertação (Mestrado em Tecnologia de Alimentos) - Universidade Federal do Ceará. Departamento de Tecnologia de Alimentos, Fortaleza.

OLIVEIRA, A. C.; VALENTIM, I. B.; GOULART, M. O. F.; SILVA, C. A.; BECHARA, E. J. H.; TREVISAN, M. T. S. Fontes vegetais naturais de antioxidantes. Química Nova, São Paulo, v. 32, n. 3, p. 689-702, 2009.
PEARSON, D. Tecnicas de laboratorio para el analises de alimentos. Zaragoza: Editorial Acribia, 1976.

PEREIRA, A. C. S.; SIQUEIRA, A. M. A.; FARIAS, J. M.; MAIA, G. A.; FIGUEIREDO, R. W.; SOUSA, P. H. M. Desenvolvimento de bebida mista à base de água de coco, polpa de abacaxi e acerola. Archivos Latinoamericanos de Nutricion, Venezuela, v. 59, n. 4, p. 441-447, 2009.

PEREIRA, C. Q.; LAVINAS, F. C.; LOPES, M. L. M.; VALENTE-MESQUITA, V. L. Industrialized cashew juices: variation of ascorbic acid and other physicalchemical parameters. Ciência e Tecnologia de Alimentos, Campinas, v. 28, p. 266-270, 2008. Suplemento.

REICHER, F.; SIERAKOWSKI, M. R.; CORRÊA, J. B. C. Determinação espectrofotométrica de taninos pelo reativo, fosfotúngstico-fosfomomolíbdico. Arquivos de Biologia e Tecnologia, Curitiba, v. 24, n. 4, p. 401-411, 1981.

RIBEIRO, S. M. R.; QUEIROZ, J. H.; QUEIROZ, M. E. L. R.; CAMPOS, F. M.; SANT'ANA, H. M. P. Antioxidant in Mango (Mangifera indica L.) Pulp. Plant Foods for Human Nutrition, Long Island, v. 62, n. 1, p. 13-17, 2007.

SANTOS, C.A. A.; COELHO, A. F. S.; CARREIRO, S. C. Avaliação microbiológica de polpas de frutas congeladas. Ciência e Tecnologia de Alimentos, Campinas, v. 28, n. 4, p. 913-915, 2008.

SCHERER, R.; RYBKA, A. C. P.; GODOY, H. P. Determinação simultânea dos ácidos orgânicos tartárico, málico, ascórbico e cítrico em polpas de acerola, caju e açaí e avaliação da estabilidade em sucos de caju. Química Nova, São Paulo, v. 31, n. 5, p. 1137-1140, 2008.

SILVA, F. C. da; GUIMARAES, D. H. P.; GASPARETTO, C.A. Reologia do suco de acerola: efeitos da concentração e temperatura. Ciência e Tecnologia de Alimentos, Campinas, v. 25, n. 1, p. 121-126, 2005.

SILVA, N.; JUNQUEIRA, V. C. A.; SILVEIRA, N. F. A. Manual de métodos de análise microbiológica de alimentos. 2. ed. São Paulo: Livraria Varela, 2001. 229 p.

SILVA, W. S. Qualidade e atividade antioxidante em frutos de variedades de aceroleira. 2008. Dissertação (Mestrado em Tecnologia em Alimentos) - Universidade Federal do Ceará. Centro de Ciências Agrárias, Fortaleza.

SOUSA, P. H. M.; MAIA, G. A.; AZEREDO, H. M. C.; SOUSA FILHO, M de S. M.; GARRUTI, D. S.; 
FREITAS, C. A. S. Mixed tropical fruit nectars with added energy components. International Journal of Food Science \& Technology, Manchester, v. 42, n. 11, p. 12901296, 2007.

SUGAI, A. Y. Processamento descontínuo de purê de manga (Mangifera indica Linn.), variedade Haden: estudo da viabilidade do produto para pronto consumo. 2002. Dissertação de Mestrado (Mestre em Engenharia) - Departamento de Engenharia Química da Escola Politécnica da Universidade de São Paulo. Universidade de São Paulo, São Paulo.
VIDAL, J. R. M. B. Estudo reológico do suco de manga - efeito dos sólidos insolúveis. 1997. Tese (Mestrado Engenharia de Alimentos) - Universidade Estadual de Campinas. FEA/UNICAMP, Campinas.

VIDAL, J. R. M. B.; SIERAKOWSKI, M. R.; HAMINIUK, C. W. I.; MASSON, M. L. Propriedades reológicas da polpa de manga (Mangifera indica L. cv. Keitt) centrifugada. Ciênc. Agrotec., Lavras, v. 3, n. 5, p. 955-960, 2006.

YAMASHITA, F.; BENASSI, M. T.; TONZAR, A. C. Produtos de acerola: estudo da estabilidade de vitamina C. Ciência e Tecnologia de Alimentos, Campinas, v. 23, n. 1, p. 92-94, 2003. 
